\title{
Rapid increase of Plasmodium falciparum dhfr/dhps resistant haplotypes, after the adoption of sulphadoxine-pyrimethamine as first line treatment in 2002, in southern Mozambique Sonia Enosse*1,2, Pascal Magnussen³, Fatima Abacassamo², Xavier Gómez- Olivé $^{2,4}$, Anita M Rønn ${ }^{5}$, Ricardo Thompson ${ }^{1,2}$ and Michael Alifrangis ${ }^{5}$
}

\begin{abstract}
Address: ${ }^{1}$ Instituto Nacional de Saúde, Ministério de Saúde, Maputo, Mozambique, ${ }^{2}$ Centro de Investigação em Saúde da Manhiça, Ministério de Saúde, Maputo, Mozambique, ${ }^{3}$ DBL-Centre of Health Research and Development, Faculty of Life Sciences, Copenhagen University, Denmark, ${ }^{4} \mathrm{MRC} /$ Wits University, Rural Public Health and Health Transitions Research Unit (Agincourt), School of Public Health, University of the Witwatersrand, South Africa and ${ }^{5}$ Centre for Medical Parasitology at Department of International Health, Immunology and Microbiology, University of Copenhagen and at Department of Infectious Diseases, Copenhagen University Hospital (Rigshospitalet), Denmark
\end{abstract}

Email: Sonia Enosse* - Senosse@teledata.mz; Pascal Magnussen - Pma@life.ku.dk; Fatima Abacassamo - Fabacassamo@yahoo.com; Xavier Gómez-Olivé - Xavier@agincourt.co.za; Anita M Rønn - Alifrangis@cmp.dk; Ricardo Thompson - Rthompson@misau.gov.mz; Michael Alifrangis - Micali@sund.ku.dk

* Corresponding author

Published: I July 2008

Malaria Journal 2008, 7:115 doi:10.1186/1475-2875-7-115
Received: 27 February 2008

Accepted: I July 2008

This article is available from: http://www.malariajournal.com/content/7/I/II5

(c) 2008 Enosse et al; licensee BioMed Central Ltd.

This is an Open Access article distributed under the terms of the Creative Commons Attribution License (http://creativecommons.org/licenses/by/2.0), which permits unrestricted use, distribution, and reproduction in any medium, provided the original work is properly cited.

\begin{abstract}
Background: In late 2002, the health authorities of Mozambique implemented sulphadoxine-pyrimethamine (SP)/amodiaquine (AQ) as first-line treatment against uncomplicated falciparum malaria. In 2004, this has been altered to SP/artesunate in line with WHO recommendations of using Artemisinin Combination Therapies (ACTs), despite the fact that all the neighbouring countries have abandoned SP-drug combinations due to high levels of SP drug resistance. In the study area, one year prior to the change to SP/AQ, SP alone was used to treat uncomplicated malaria cases. The study described here investigated the immediate impact of the change to SP on the frequency of SP and CQ resistance-related haplotypes in the Plasmodium falciparum genes Pfdhfr, Pfdhps and Pfcrt before and a year after the introduction of SP.

Methods: Samples were collected during two cross sectional surveys in early 2002 and 2003 involving 796 and 692 children one year or older and adults randomly selected living in Maciana, an area located in Manhiça district, Southern Mozambique. Out of these, I7I and I73 P. falciparum positive samples were randomly selected to measure the frequency of resistance- related haplotypes in Pfdhfr, Pfdhps and Pfcrt based on results obtained by nested PCR followed by sequence-specific oligonucleotide probe (SSOP)-ELISA.

Results: The frequency of the SP-resistance associated Pfdhps double mutant (SGEAA) haplotype increased significantly from $14 \%$ to $35 \%(\mathrm{P}<0.00 \mathrm{I})$, while the triple mutant $\mathrm{Pfdhfr}$ haplotype $(\mathrm{CIRNI})$ remained high and only changed marginally from $46 \%$ to $53 \%(P=0.405)$ after one year with $S P$ as first-line treatment in the study area. Conversely, the combined Pfdhfr/Pfdhps quintuple mutant haplotype increased from $8 \%$ to $26 \%(P=0.005)$. The frequency of the chloroquine resistance associated Pfcrt-CVIET haplotype was above $90 \%$ in both years.

Conclusion: These retrospective findings add to the general concern on the lifespan of the combination of SP/ artesunate in Mozambique. The high frequency of quintuple Pfdhfr/Pfdhps haplotypes found here as early as 2002 most likely cause ideal conditions for the development of artesunate tolerance in the $P$. falciparum populations and may even endanger the sensitivity to the second-line drug, Coartem ${ }^{\circledR}$.
\end{abstract}




\section{Background}

Large scale prevalence of Plasmodium falciparum chloroquine (CQ), amodiaquine (AQ) and sulphadoxine/ pyrimethamine (SP) resistance has forced countries with endemic malaria to change to the artemisinin combination therapies (ACTs) as first-line treatment against uncomplicated malaria. The ACT drugs, currently being implemented in sub-Saharan Africa combine various artemisinin analogues with novel drugs (lumefantrine, i.e. Coartem ${ }^{\circledast}$ ) or with already widely used drugs such as AQ and SP. ACTs are highly efficacious and are likely to decrease the rate of development of resistance, and thus expand the useful lifetime of the drugs [1]. Artemisinin derivates are acting and are eliminated rapidly after treatment and the development of resistance to ACTs will probably depend on the already existing background level of resistance to the partner drugs in the parasite population, as it has been observed in areas with high levels of resistance to SP and AQ [2,3]. Currently, SP combined with artesunate (ART) is the first-line treatment policy in few African countries, such as Mozambique (from 2004) and Northern Sudan [4,5]. In Mozambique, prior to adoption of the ACT strategy, the combination of AQ and SP as the first-line treatment was implemented in late 2002 following reports of high levels of chloroquine (CQ) resistance [6]. However, one year prior to this change, SP alone was used to treat uncomplicated malaria cases in the study area. Additionally, SP has recently been used in programmes examining the efficacy of intermittent presumptive treatment (IPT) intervention in pregnant women and infants in Mozambique [7,8].

The prevalence of combinations of single nucleotide polymorphisms (SNP) in the P. falciparum dihydrofolate reductase (Pfdhfr) and dihydropteroate synthetase (Pfdhps) genes have been correlated with resistance to SP in vivo $[9,10]$. In Africa, the Pfdhfr triple mutation, $\mathrm{N} 51 \mathrm{I}+\mathrm{C} 59 \mathrm{R}+\mathrm{S} 108 \mathrm{~N}$, with wildtype at codons 50 (C50) and 164 (I164), is combined into a highly prevalent and resistant haplotype, CIRNI. For Pfdhps the double mutation, A437G + K540E, with wildtype at codons 436 (S436) 581 (A581) and 613 (A613) are combined into the resistant haplotype SGEAA. The combination of the two; CIRNI-SGEAA, have been suggested as a molecular marker of in vivo resistance [10]. However, recent studies have questioned the association between this combination of molecular markers and drug resistance in vivo by showing that differences in responses to SP treatment was a consequence of acquired immunity rather than parasite factors $[11,12]$.

Resistance to both CQ and AQ is mainly associated with a single K76T mutation in the P. falciparum chloroquine resistance transporter gene (Pfcrt), but are probably involving mutations in the $P$. falciparum multi-drug resistance (Pfmdr1) gene as well $[13,14]$. Three main haplotypes in codons 72-76 of the Pfcrt gene exist, namely the wild type CVMNK and the CQ resistant haplotypes, CVIET and SVMNT [15], where CVIET is by far the most dominant mutant haplotype in Africa while SVMNT haplotypes has only been observed sporadically [16].

The objective of this study was to determine the frequency of Pfdhfr, Pfdhps and Pfcrt resistance related haplotypes in $P$. falciparum isolates collected before and a year after SP was introduced as first-line treatment, as replacement for CQ in the study area. Moreover, since Mozambique was implementing a treatment policy with SP in combination with artesunate as first line treatment of uncomplicated malaria, the findings of this study in addition to recent studies $[17,18]$ is providing crucial baseline information of the level of drug resistance related haplotypes present in the parasite population prior to the adoption of the ACT strategy in the country.

\section{Methods}

\section{Study population and samples}

The study was conducted in Maciana, a village located in Manhiça district Southern Mozambique with 14.496 inhabitants [19]. The climate is characterized by a warm and wet season from November to April and a dry and cold season from May to October. The area is hyperendemic for malaria and the transmission is perennial with marked seasonality and the majority of cases occur during the rainy season reaching a peak in during February and May. Ninety percent of all infections are caused by $P$. falciparum, but $P$. malariae and $P$. ovale are also observed. The population is mainly made up of farmers growing maize and beans and do have access to one health post which serves only outpatients [19]. Samples for analysis of molecular markers of drug resistance were collected during two cross sectional surveys involving 796 and 692 children one year or older and adults randomly selected from the Manhiça demographic surveillance system. The first cross-sectional study was conducted before the introduction of SP as first-line treatment in the study area, from December 2001 to January 2002 (survey 1); and the second, from January to February 2003 (survey 2). Finger prick blood samples were obtained for thick and thin blood smears and for filter paper blood spots.

Ethical clearance for the study was provided by the National Institute of Health, Ministry of Health of Mozambique Ethical Committee. The Danish National Committee on Biomedical Research Ethics commented on the proposal and recommended the study. Written informed consent was obtained from adults and the parents/guardians of the children before enrolment. 


\section{DNA extraction and detection of polymorphisms in of Pfdhfr, Pfdhps and the Pfcrt}

For practical reasons, 171 and 173 samples from cross sectional survey 1 and 2, respectively, were selected randomly for the analyses of polymorphisms in Pfdhfr, Pfdhps and Pfcrt. DNA extraction from bloodspots and nested PCR followed by the sequence specific oligonucleotide probe (SSOP)-ELISA was performed and analysed as previously described [20] using SSOPs targeting SNPs in Pfd$h f r$ at c50/51 (CI/CN), c59 (C/R), c108 (S/N/T) and c164 (I/L) and in Pfdhps at c436/437 (AA/AG/SA/SG/FG) c540 $(\mathrm{K} / \mathrm{E}), \mathrm{c} 581(\mathrm{~A} / \mathrm{G})$ and $\mathrm{c} 613(\mathrm{~A} / \mathrm{S})$. The resulting single nucleotide polymorphisms (SNPs) were constructed into haplotypes. For Pfcrt, SSOPs targeting the haplotypes CVMNK, CVIET and SVMNT was used [20].

\section{Statistics}

All statistical analyses were performed using SigmaStat software package version 3.0.1 and SPSS statistical package version 15.0. For the statistical analyses of the characteristics of the individuals sampled during the crosssectional studies, the differences between groups were assessed using the Chi-square test for proportions and the Student t-test for continuous normal distributed variables. Frequencies of mutations between groups were compared using the Chi-square or Fisher exact test. In addition, a logistic regression model was used to compare haplotype distributions obtained in the two cross-sectional surveys while adjusting for the potential confounding effects of $P$. falciparum density and age.

\section{Results}

A total of 244 and 192 samples were $P$. falciparum positive by microscopy in cross-sectional survey 1 and 2, respectively out of which 171 and 173 samples were chosen randomly for the genotyping analysis. The populations in the two surveys differed in respect to age and parasite density: In survey 2 , the median age was significantly lower $(\mathrm{P}=$ $0.013)$ while the parasite density was significantly higher $(\mathrm{P}<0.001)$ compared to survey 1 (Table 1$)$. Only Pfdhfr wildtypes at c164 was found, while for Pfdhps, only wildtypes were observed at c518 and c613.
A significant increase in the frequency of Pfdhps double mutant haplotype, SGEAA from $13.5 \%$ to $34.8 \%$ over a year was found $\left(\chi^{2}=12.24, \mathrm{P}<0.001\right)$, mainly decreasing the frequency of Pfdhps wildtypes SAKAA/AAKAA from $80.8 \%$ to $64.3 \%\left(\chi^{2}=5.893, P=0.015\right.$; Figure $\left.1 \mathrm{~A}\right)$. For Pfdhfr, there was only a marginal difference between the two surveys with a frequency of the triple mutant haplotype, CIRNI at $46.5 \%$ and $53.2 \%$ in survey 1 and 2, respectively $\left(\chi^{2}=0.70, P=0.405\right.$; Figure $\left.1 \mathrm{~B}\right)$. When combining the two loci, the frequency of parasites carrying the highly resistant $P f d h f r / P f d h p s$ quintuple haplotype increased from $8.0 \%$ to $25.8 \%\left(\chi^{2}=7.78, P=0.005\right)$ between survey 1 and 2 . The change in frequency was not at the expense of the wildtype Pfdhfr/Pfdhps haplotype, CNCSI-SAKAA that remained stable at $11 \%$, but rather on the double mutant haplotypes, where the CNRNI-SAKAA accounted for a decrease from $26.7 \%$ to $18.0 \%\left(\chi^{2}=1.32, \mathrm{P}=0.250\right)$ and the triple mutant haplotype, mainly the CIRNISAKAA that decreased from $37.3 \%$ to $27.0 \%\left(\chi^{2}=1.57, \mathrm{P}\right.$ $=0.210$ ) (Figure 2). Logistic regression analysis revealed that all of the above mentioned statistical results (with regards to significance) persisted after appropriate adjustment for $P$. falciparum density and age.

The prevalence of single nucleotide polymorphisms in Pfdhfr and Pfdhps is shown in Table 2. In Pfdhfr, only minor differences can be seen between the two cross sectional surveys where a strong trend for a decrease in the prevalence of the 108S wildtype is apparent (from $49.6 \%$ to $\left.38.0 \%, \chi^{2}=3.06, \mathrm{P}=0.080\right)$. For Pfdhps, as expected from the combined haplotype data, both a decrease of the 436/437SA wildtype (from $85.7 \%$ to $71.0 \%, \chi^{2}=7.18, \mathrm{P}$ $=0.007)$ and an increase in the mutant 436/437SG mutant type (from $56.4 \%$ to $68.5 \%, \chi^{2}=3.46, \mathrm{P}=0.063$ ) is evident. Furthermore, the prevalence of the $540 \mathrm{~K}$ wildtype is decreasing significantly from $92.1 \%$ to $71.6 \%$ $\left(\chi^{2}=16.66, \mathrm{P}<0.001\right)$.

Major differences in prevalence of infections with Pfdhps and combined Pfdhfr/Pfdhps mutations were found when comparing different age groups (i.e. 1-5, 6-14 and $>14$ years), however only in the second survey (Table 3): The

Table I: Characteristics of the randomly selected samples for the analysis of molecular markers of drug resistance from cross sectional surveys in December 200 I (CrossI) and January 2003 (Cross 2)

\begin{tabular}{|c|c|c|c|}
\hline & $\begin{array}{c}\text { Cross } 1 \\
N=|7|\end{array}$ & $\begin{array}{c}\text { Cross2 } \\
N=173\end{array}$ & P-value \\
\hline Median age (years) (25-75\% percentile) & $\begin{array}{c}14 \\
(8.0-27.8)\end{array}$ & $\begin{array}{c}11 \\
(6.0-23.5)\end{array}$ & 0.013 \\
\hline Gender, male (\%) & 43.3 & 45.7 & 0.772 \\
\hline Median P. falciparum density, parasites/ $\mu \mathrm{l}$ (25-75\% percentile) & $\begin{array}{c}125 \\
(63-423)\end{array}$ & $\begin{array}{c}332 \\
(84-2242)\end{array}$ & $<0.001$ \\
\hline Donors with temperature $>37.5^{\circ} \mathrm{C}(\%)$ & 4.1 & 4.6 & 0.982 \\
\hline
\end{tabular}

P-values: Mann-Whitney Rank Sum Test or $\chi^{2}$-tests comparisons between the two cross sectional surveys. 

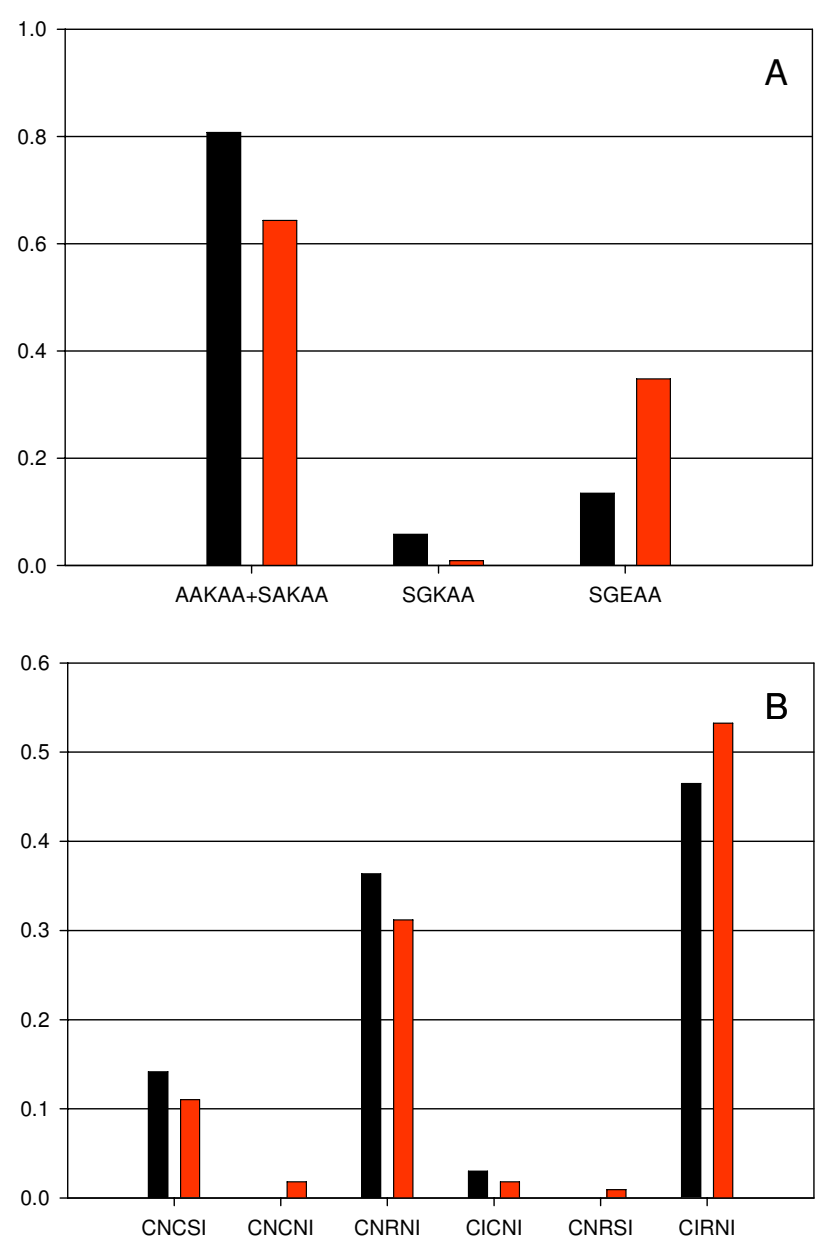

Figure I

The Frequency of Pfdhps (A) and Pfdhfr (B) haplotypes in December 200 I (black bars) and January 2003 (red bars) in Manhiça district. After removing the PCR negatives and the samples with mixed haplotype infections (where a major haplotype could not be determined), for Pfdhps, $\mathrm{N}=104$ and II 5 and for Pfdhfr, $\mathrm{N}=99$ and 109 from survey $I$ and 2 , respectively.

prevalence of quintuple mutant haplotype was significantly higher in children under fives compared to the older age groups $\left(\chi^{2}=6.04, \mathrm{P}=0.049\right)$ and a strong tendency for a higher frequency of Pfdhps double mutant haplotypes in children under fives compared to the older groups was seen $\left(\chi^{2}=5.33, P=0.069\right)$.

The samples analysis of the Pfcrt gene showed almost exclusively the occurrence of the mutant haplotype CVIET in both years (92.8\% in survey 1 and $95.3 \%$ in survey 2; $\chi^{2}=0.74, \mathrm{P}=0.389$ ), while the remaining samples were the wild type CVMNK haplotype.
Table 2: The prevalence of single nucleotide polymorphisms (SNP) at codon 5I, 59 and 108 of Pfdhfr and codon $436 / 437$ and 540 of Pfdhps in the two cross sectional surveys

\begin{tabular}{|c|c|c|c|c|c|c|}
\hline \multicolumn{2}{|c|}{ Pfdhfr codon } & \multicolumn{2}{|c|}{ CrossI } & \multicolumn{2}{|c|}{ Cross2 } & \multirow[t]{2}{*}{$P$} \\
\hline & & $\mathrm{N}$ & (\%) & $\mathrm{N}$ & $(\%)$ & \\
\hline \multirow[t]{3}{*}{51} & $N$ & 43 & 32.3 & 33 & 27.8 & 0.169 \\
\hline & I & 49 & 36.8 & 58 & 46.0 & 0.507 \\
\hline & Mixed (N/I) & 41 & 30.8 & 33 & 26.2 & \\
\hline \multirow[t]{3}{*}{59} & C & 16 & 12.7 & 14 & 11.0 & 0.853 \\
\hline & $\mathrm{R}$ & 66 & 52.4 & 69 & 54.3 & 0.828 \\
\hline & Mixed $(C / R)$ & 44 & 87.3 & 44 & 34.6 & \\
\hline \multirow[t]{3}{*}{108} & $\mathrm{~S}$ & 14 & 11.0 & 8 & 6.2 & 0.080 \\
\hline & $\mathrm{N}$ & 64 & 50.4 & 80 & 62.0 & 0.249 \\
\hline & Mixed $(\mathrm{S} / \mathrm{N})$ & 49 & 38.6 & 41 & 31.8 & \\
\hline \multicolumn{2}{|c|}{ Pfdhps codon } & $\mathrm{N}$ & (\%) & $\mathrm{N}$ & $(\%)$ & \\
\hline \multirow[t]{7}{*}{$436 / 437$} & $\mathrm{AA}$ & 3 & 2.4 & I & 0.8 & 0.746 \\
\hline & SA & 50 & 39.7 & 37 & 29.8 & 0.007 \\
\hline & SG & 15 & 11.9 & 35 & 28.2 & 0.063 \\
\hline & Mixed & & & & & \\
\hline & AA/SA & 2 & 1.6 & I & 0.8 & \\
\hline & SA/SG & 56 & 44.4 & 45 & 36.3 & \\
\hline & AA/SA/SG & 0 & 0 & 5 & 4.0 & \\
\hline \multirow[t]{3}{*}{540} & $\mathrm{~K}$ & 80 & 63.5 & 73 & 54.5 & $<0.001$ \\
\hline & $E$ & 10 & 7.9 & 38 & 28.4 & 0.117 \\
\hline & Mixed (K/E) & 36 & 28.6 & 23 & 17.2 & \\
\hline
\end{tabular}

The data are given as total numbers and as percentages and including genotype infections where the identification of SNP/haplotypes for a few samples were not complete. Mixed: Prevalence of infections with mixed SNP for each codon. P: P-values of $\chi^{2}$-tests of prevalence comparisons between the two cross sectional surveys where the prevalence of infections of each SNP including mixed infections are set against prevalence of infections without the particular SNP.

\section{Discussion}

In late 2002, Mozambique changed the treatment policy from CQ to SP/AQ against uncomplicated malaria. Prior to this change, the present study implemented SP alone as first line treatment in the study area and as a possible consequence, the study observed a rapid increase in frequency of double SGEAA-Pfdhps and quintuple CIRNI-SGEAA$P f d h f r / P f d h p s$ mutant haplotypes at the community level just a year after. Prior to the introduction of SP the consumption of this drug was very low in the study area [19]. However, since the study did not measure the actual use of SP outside the health services, the study cannot rule out alternative explanations such as consumption of other antifolate drugs and for instance changes in migration. The triple mutant CIRNI-Pfdhfr haplotype, was highly prevalent prior to the change $(46.5 \%)$, also observed in 2000 in Matola (50.9\%), a peri-urban area within Maputo province [21]. Similarly, Raman et al observed a remarkable increase of the triple mutant haplotype in 13 sentinel sites stratified into three zones in Maputo province between 1999 and 2003, almost reaching 100\% in 2003 


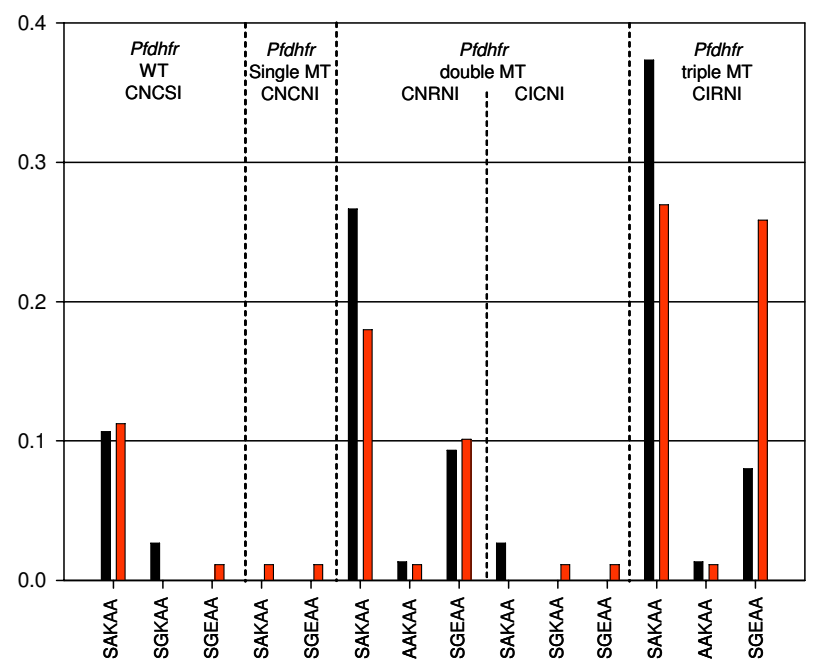

Figure 2

The Frequency of combined Pfdhfr/dhps haplotypes in December 200 I (dark bars) and January 2003 (red bars) in Manhiça district in Manhiça district. After removing the PCR negatives and the samples with mixed haplotype infections (where a major haplotype could not be determined), $\mathrm{N}=75$ and 89 samples from survey $\mathrm{I}$ and 2 , respectively.

[18], while Fernandes et al found $93 \%$ of the triple mutant haplotype in samples from children with uncomplicated malaria attending a Health Centre in Maputo city of Mozambique in 2004 [17].

This high frequency of $P f d h f r$ mutations before the actual implementation of SP/AQ as first-line drug in Mozambique may be due to the use of other chemically related drugs such as co-trimoxazole, which is commonly used in the study area for treating bacterial infections and as a preventive measure in HIV/AIDS patients (Dr Yunuss Assane, $\mathrm{MoH}$, personal communication). While not shown in vivo, in vitro cross-resistance has been reported between co-trimoxazole and SP components [22]. More importantly, as suggested by Raman et al, since neighbouring KwaZulu-Natal province in South Africa implemented SP as early as 1988, the high frequency before SP implementation in Mozambique may be caused by SP drug pressure from the border region [18].

In the present study, the most notable change was the increase of the double mutant Pfdhps SGEAA haplotype from $13.5 \%$ to $34.8 \%$ over only one year. In the study by Raman et al, the frequency of the double mutant haplotype peaked in 2001 at $19-25 \%$ in all three zones followed by a remarkable and significant decrease in 2003 and 2004 to less than $10 \%$ [18], in sharp contrast to the findings by Fernandes et al, where the frequency was 51\%
Table 3: The prevalence of triple mutations in Pfdhfr, double mutations in Pfdhps and quintuple mutations in Pfdhfr/Pfdhps in the two cross sectional surveys according to age

\begin{tabular}{|c|c|c|c|c|c|c|}
\hline \multirow[b]{2}{*}{$\begin{array}{l}\text { Age } \\
\text { (years) }\end{array}$} & \multicolumn{2}{|c|}{ Pfdhfr triple MT } & \multicolumn{2}{|c|}{ Pfdhps double MT } & \multicolumn{2}{|c|}{$\begin{array}{l}\text { Pfdhfr and Pfdhps } \\
\text { quintuple MT }\end{array}$} \\
\hline & CrossI & Cross2 & CrossI & Cross2 & Cross I & Cross 2 \\
\hline $1-5$ & $\begin{array}{c}4 / 12 \\
(33.3)\end{array}$ & $\begin{array}{l}16 / 24 \\
(66.7)\end{array}$ & $\begin{array}{l}2 / 12 \\
(16.7)\end{array}$ & $\begin{array}{l}|1 / 2| \\
(52.4)\end{array}$ & $\begin{array}{l}1 / 10 \\
(10.0)\end{array}$ & $\begin{array}{l}8 / 16 \\
(50.0)\end{array}$ \\
\hline $6-14$ & $\begin{array}{l}20 / 43 \\
(46.5)\end{array}$ & $\begin{array}{l}24 / 43 \\
(55.8)\end{array}$ & $\begin{array}{l}7 / 46 \\
(15.2)\end{array}$ & $\begin{array}{l}17 / 45 \\
(37.8)\end{array}$ & $\begin{array}{l}1 / 31 \\
(3.2)\end{array}$ & $\begin{array}{l}8 / 36 \\
(22.2)\end{array}$ \\
\hline$>14$ & $\begin{array}{l}22 / 44 \\
(50.0)\end{array}$ & $\begin{array}{l}18 / 42 \\
(42.9)\end{array}$ & $\begin{array}{l}7 / 46 \\
(15.2)\end{array}$ & $\begin{array}{l}12 / 49 \\
(24.5)\end{array}$ & $\begin{array}{c}4 / 34 \\
(11.8)\end{array}$ & $\begin{array}{l}7 / 37 \\
(18.9)\end{array}$ \\
\hline$P=$ & 0.591 & 0.160 & 0.991 & 0.069 & 0.434 & 0.049 \\
\hline
\end{tabular}

The donors are divided into I-5 years, 6-14 years and above 14 years of age. Actual numbers of infections with Pfdhfr triple, Pfdhps double and Pfdhfr/Pfdhps quintuple mutations are shown out of total with the percentage of total in brackets. P: P-value of $\chi^{2}$-tests.

in their 2004 study [17], presumably due to differences in sampling where the latter study was done on samples from symptomatic children attending a health centre. Such a difference in frequency in symptomatic cases versus asymptomatic carriers has likewise been shown in a study performed in Tanzania [23].

In the second survey of this study, the quintuple Pfdhfr/ Pfdhps haplotype was more frequent in children under fives compared to the older age groups. Similar findings, but rather for $P f d h f r$ haplotypes alone was seen by Raman et al [18] and is most likely due to a stronger survival advantage of the mutant parasites in the younger age groups due to the more frequent treatments this group receive. It also pinpoints a potential pitfall when measuring prevalence of molecular markers of drug resistance in under-fives when these data are extrapolated to cover the population as a whole.

The high prevalence of these mutant haplotypes in the area as early as 2001 along with the findings by Fernandes et al [17] and Raman et al [18], questions the current efficacy of the first-line antimalarial drug in Mozambique, a combination of ART and SP. Results from in vivo study carried out in the same area in 2001 have described a relatively high adequate clinical response to SP after 14 days of follow-up at $82.7 \%$ [6]. However, the levels of treatment failures and RI and RII parasitological resistance increased when follow-up was extended to 28 days, indicating a decline in the efficacy of SP as early as 2001. Most likely, initially ART/SP was an efficient treatment against uncomplicated malaria in Mozambique, however the risk of clinical treatment failures could be expected to appear within a short span of years, since the benefits of ACT 
combinations are diminished by the inclusion of an ineffective partner drug, such as SP, where ART/SP may be equivalent to ART monotherapy. Despite that mutations in $P f d h f r / P f d h p s$ may not directly cause clinical ART/SP treatment failures, a high prevalence of SP resistancerelated mutations may rather indicate a risk of emerging tolerance to ART endangering the whole ACT strategy in the region. Close and frequent monitoring of the efficacy of the drug combination should be performed as well as formulation of a strategy for alternative first line treatment.

\section{Conclusion}

The high frequency of quintuple $P f d h f r / P f d h p s$ haplotypes found here as early as 2002 most likely cause ideal conditions for the development of ART tolerance in the P. falciparum populations and may even endanger the sensitivity to the second-line drug, Coartem.

\section{Authors' contributions}

SE, PM, AMR, RT and MA conceived and designed the study. SE, FA, FXG-O and RT was responsible for the cross sectional sampling. SE performed the experiments and analyses with MA. SE, FXG-O, PM, FA, RT and MA participated in manuscript preparation. All authors read and approved the final manuscript.

\section{Acknowledgements}

We thank the residents of Maciana for their participation in this study and the field workers and laboratory technician from CISM for their technical assistance in the study. We are gratefully to Dr. Pedro Alonso, at CISM, for advice during the field work and his helpful comments on the manuscript. We acknowledge laboratory technician Jimmy Weng at the Centre for Medical Parasitology at the University of Copenhagen for assistance with PCR and SSOP-ELISA. Dr. Henry Madsen at DBL-Institute of Health Research and Development and Dan W. Meyrowitsch at the Institute of Public Health at the University of Copenhagen are acknowledged for statistical assistance.

\section{References}

I. White $\mathrm{N}$ : Antimalarial drug resistance and combination chemotherapy. Philos Trans R Soc Lond B Biol Sci 1999, 354:739-749.

2. Mutabingwa TK, Anthony D, Heller A, Hallett R, Ahmed J, Drakeley C, Greenwood BM, Whitty CJ: Amodiaquine alone, amodiaquine+sulfadoxine-pyrimethamine, amodiaquine+artesunate, and artemether-lumefantrine for outpatient treatment of malaria in Tanzanian children: a four-arm randomised effectiveness trial. Lancet 2005, 365: I474-I480.

3. Rwagacondo CE, Niyitegeka F, Sarushi J, Karema C, Mugisha V, Dujardin JC, Van Overmeir C, van den EJ, D'Alessandro U: Efficacy of amodiaquine alone and combined with sulfadoxinepyrimethamine and of sulfadoxine pyrimethamine combined with artesunate. Am J Trop Med Hyg 2003, 68:743-747.

4. Mutabingwa TK: Artemisinin-based combination therapies (ACTs): Best hope for malaria treatment but inaccessible to the needy! Acta Trop 2005, 95:305-3I5.

5. Tiago A, Saute F: Normas de Manejo de casos de malária em Mozambique. Miniserio da Saude, Programa Nacional de Controlo da Malaria. Miniserio da Saude, Programa Nacional de Controlo da Malaria; 2005.

6. Abacassamo F, Enosse S, Aponte JJ, Gomez-Olive FX, Quinto L, Mabunda S, Barreto A, Magnussen P, Ronn AM, Thompson R, Alonso $\mathrm{PL}$ : Efficacy of chloroquine, amodiaquine, sulphadoxine- pyrimethamine and combination therapy with artesunate in Mozambican children with non-complicated malaria. Trop Med Int Health 2004, 9:200-208.

7. Macete E, Aide P, Aponte J], Sanz S, Mandomando I, Espasa M, Sigauque B, Dobano C, Mabunda S, Dgedge M, Alonso P, Menendez C: Intermittent preventive treatment for malaria control administered at the time of routine vaccinations in Mozambican infants: a randomized, placebo-controlled trial. J Infect Dis 2006, 194:276-285.

8. Brentlinger PE, Dgedge M, Correia MA, Rojas AJ, Saute F, GimbelSherr KH, Stubbs BA, Mercer MA, Gloyd S: Intermittent preventive treatment of malaria during pregnancy in central Mozambique. Bull World Health Organ 2007, 85:873-879.

9. Kyabayinze D, Cattamanchi A, Kamya MR, Rosenthal PJ, Dorsey G: Validation of a simplified method for using molecular markers to predict sulfadoxine-pyrimethamine treatment failure in African children with falciparum malaria. Am J Trop Med Hyg 2003, 69:247-252.

10. Kublin JG, Dzinjalamala FK, Kamwendo DD, Malkin EM, Cortese JF, Martino LM, Mukadam RA, Rogerson SI, Lescano AG, Molyneux ME, Winstanley PA, Chimpeni P, Taylor TE, Plowe CV: Molecular markers for failure of sulfadoxine-pyrimethamine and chlorproguanil-dapsone treatment of Plasmodium falciparum malaria. J Infect Dis 2002, 185:380-388.

I I. Francis D, Nsobya SL, Talisuna A, Yeka A, Kamya MR, Machekano R, Dokomajilar C, Rosenthal PJ, Dorsey G: Geographic differences in antimalarial drug efficacy in Uganda are explained by differences in endemicity and not by known molecular markers of drug resistance. J Infect Dis 2006, 193:978-986.

12. Enevold A, Nkya WM, Theisen M, Vestergaard LS, Jensen AT, Staalsoe T, Theander TG, Bygbjerg IC, Alifrangis M: Potential impact of host immunity on malaria treatment outcome in Tanzanian children infected with Plasmodium falciparum. Malar J 2007, 6:153.

13. Holmgren G, Gil JP, Ferreira PM, Veiga MI, Obonyo CO, Bjorkman A: Amodiaquine resistant Plasmodium falciparum malaria in vivo is associated with selection of pfcrt $76 \mathrm{~T}$ and pfmdr I $86 \mathrm{Y}$. Infect Genet Evol 2005.

14. Happi CT, Gbotosho GO, Folarin OA, Bolaji OM, Sowunmi A, Kyle DE, Milhous W, Wirth DF, Oduola AM: Association between mutations in Plasmodium falciparum chloroquine resistance transporter and P. falciparum multidrug resistance I genes and in vivo amodiaquine resistance in $\mathbf{P}$. falciparum malariainfected children in Nigeria. Am J Trop Med Hyg 2006, 75: $155-161$

15. Warhurst DC: Polymorphism in the Plasmodium falciparum chloroquine-resistance transporter protein links verapamil enhancement of chloroquine sensitivity with the clinical efficacy of amodiaquine. Malar J 2003, 2:31.

16. Alifrangis M, Dalgaard MB, Lusingu JP, Vestergaard LS, Staalsoe T, Jensen AT, enevold A, Ronn AM, Khalil IF, Warhurst DC, Lemnge MM, Theander TG, Bygbjerg IC: Occurrence of the Southeast Asian/South American SVMNT haplotype of the chloroquine-resistance transporter gene in Plasmodium falciparum in Tanzania. J Infect Dis 2006, I93:1738-I74I.

17. Fernandes N, Figueiredo P, do R V, Cravo P: Analysis of sulphadoxine/pyrimethamine resistance-conferring mutations of Plasmodium falciparum from Mozambique reveals the absence of the dihydrofolate reductase I64L mutant. Malar J 2007, 6:35.

18. Raman J, Sharp B, Kleinschmidt I, Roper C, Streat E, Kelly V, Barnes KI: Differential Effect of Regional Drug Pressure on Dihydrofolate Reductase and Dihydropteroate Synthetase Mutations in Southern Mozambique. Am J Trop Med Hyg 2008, 78:256-26I.

19. Enosse SMM: Antimalarial drug resistance in southern Mozambique: treatment efficacy and molecular characterization of Plasmodium falcipraum resistnace to sulfadoxinepyrimethamine. Instituto Nacional de Saúde; Ministério da Saude, Maputo, Mozambique. Danish Bilharziasis Laboratory, Denmark; 2004.

20. Alifrangis M, Enosse S, Pearce R, Drakeley C, Roper C, Khalil IF, Nkya WM, Ronn AM, Theander TG, Bygbjerg IC: A simple highthroughput method to detect Plasmodium falciparum single nucleotide polymorphisms in the dihydrofolate reductase, dihydropteroate synthase, and $P$. falciparum cloroquine 
resistance transporter genes using ploymerase chain reaction and enzyme linked immunosorbent assay-based technology. Am J Trop Med Hyg 2005, 72: I55-162.

21. Alifrangis M, Enosse S, Khalil IF, Tarimo DS, Lemnge MM, Thompson R, Bygbjerg IC, Ronn AM: Prediction of Plasmodium falciparum resistance to sulfadoxine/pyrimethamine in vivo by mutations in the dihydrofolate reductase and dihydropteroate synthetase genes: a comparative study between sites of differing endemicity. Am J Trop Med Hyg 2003, 69:60I-606.

22. Khalil I, Ronn AM, Alifrangis M, Gabar HA, Satti GM, Bygbjerg IC: Dihydrofolate reductase and dihydropteroate synthase genotypes associated with in vitro resistance of Plasmodium falciparum to pyrimethamine, trimethoprim, sulfadoxine, and sulfamethoxazole. Am J Trop Med Hyg 2003, 68:586-589.

23. Roper C, Pearce R, Bredenkamp B, Gumede J, Drakeley C, Mosha F, Chandramohan D, Sharp B: Antifolate antimalarial resistance in southeast Africa: a population-based analysis. Lancet 2003, 36I: II74-II8I.

Publish with Bio Med Central and every scientist can read your work free of charge

"BioMed Central will be the most significant development for disseminating the results of biomedical research in our lifetime. "

Sir Paul Nurse, Cancer Research UK

Your research papers will be:

- available free of charge to the entire biomedical community

- peer reviewed and published immediately upon acceptance

- cited in PubMed and archived on PubMed Central

- yours - you keep the copyright 